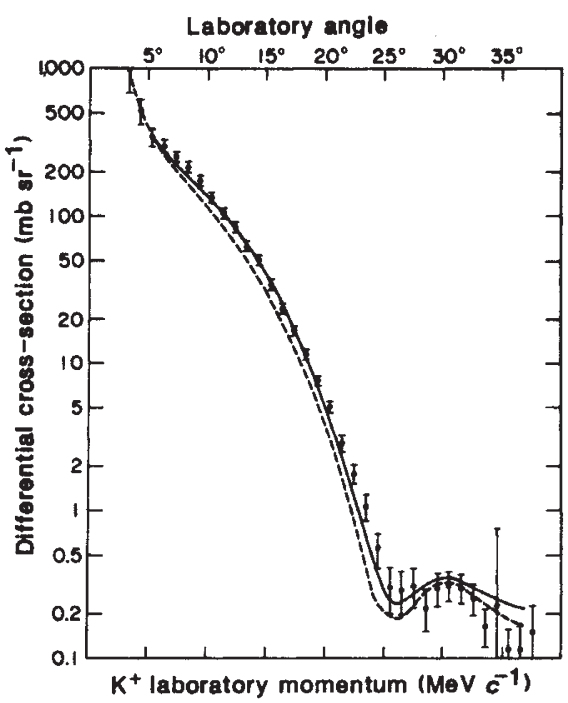

Fig. 2 Comparison with the data of Marlow et al. ${ }^{+}$of theoretical calculations of $\mathrm{K}^{+}$elastic scattering from carbon before $(\lambda=0$, broken line $)$ and after ( $\lambda=0.2$, solid line) allowing for a possible change in the size of the nucleon.

The decrease in the vector meson mass is easily understood. In a simple quark model, the $\varrho$ and $\omega$ are made of a loosely bound quark-antiquark pair. Because they are weakly bound, $m_{v}$ is approximately twice the mass of each quark $\left(2 m_{\mathrm{q}}\right)$. At very high densities, say five to ten times the density of the most dense nucleus, quantum chromodynamics (the most widely accepted theory of the strong interaction) predicts a transition to a phase in which $m_{\mathrm{q}}$ is zero. In one widely used model ${ }^{6}$ the variation of $m_{\mathrm{q}}$ between the low- and high-density limits is approximately linear ${ }^{7}$, naturally giving a 10-20 per cent decrease in $m_{v}$ at normal nuclear matter density.

Of course Fig. 2 shows calculations for $\mathrm{K}^{+}$scattering from a nucleus, not a nucleon. Can one be sure that there is no theoretical problem in computing the $\mathrm{K}^{+}-$ nucleus interaction? Although Brown et $a l$. note that there are some corrections still to be calculated, the answer is probably yes. For many years, nuclear physicists have known that the $\mathrm{K}^{+}$is an ideal probe of nuclear structure - a 'strongly interacting electron'. The reason is that the quark structure of the $\mathrm{K}^{+}$(it contains an antistrange quark) does not allow it to resonate with a nucleon in the way that (say) the related pion does. In consequence the $\mathrm{K}^{+}$-nucleon cross-section is small and varies slowly with energy. With a mean free path of typically $5 \mathrm{fm}$ or greater, a $\mathrm{K}^{+}$will rarely suffer more than one collision with a nucleon in passing through a carbon nucleus. This limited number of collisions gives one great confidence in the theoretical calculations of the $\mathrm{K}^{+}$- nucleus interaction (the microscopic optical model).

The lack of $\mathrm{K}^{+}$experimental data is attributable not to any lack of enthusiasm but to the difficulties of making usable beams. Like the pion, the $\mathrm{K}^{+}$only lives $10^{-8}$ seconds before decaying into pions which then contaminate the beam.

The experiment of Marlow et al. ${ }^{4}$ used the $\mathrm{K}^{+}$beam at the Brookhaven AGS (alternating gradient synchrotron) which is the best available. In addition the measurements were carefully performed. Nevertheless, there could be significant systematic errors - up to \pm 17 per cent in the absolute normalization of the data ${ }^{4}$. Only new experiments at a facility producing clean, intense $\mathrm{K}^{+}$beams will unambiguously resolve such questions. It is therefore encouraging to note the announcement of a detailed design study for a kaon factory at TRIUMF Labs in Vancouver, made by the governments of Canada and British Columbia on 21 July. Among the features at such a facility would be a $\mathrm{K}^{+}$ beam of intensity and purity orders of magnitude better than previously available.

The concept of nucleon swelling is controversial. Dunne and one of us (A.W.T.) have challenged, in the context of the data taken by the EMC, the consistency of interpreting dynamical rescaling as showing a significant increase in nucleon size ${ }^{8}$. Quite stringent limits have been placed on possible size changes by studying inelastic electron scattering". Nor is the treatment of nucleon size in terms of vector mesons universally accepted. An alternative view is that in regions of momentum transfer probed by electron and $\mathrm{K}^{+}$scattering one should directly use a quark model ${ }^{10}$. One of the most fundamental questions in nuclear physics is whether or not these two pictures are equivalent. A recent calculation of the binding energy and density of nuclear matter in an explicit quark model suggests not. Guichon ${ }^{11}$ found only a 2 per cent increase rather than the 10 per cent of Brown et al. ${ }^{5}$.

The controversy over the best description of nuclear physics at higher density is a sign of a healthy field. The existing paradigm of vector meson exchange may be reinforced or overthrown. The work of Brown et al. shows that experiments with intense, clean beams of $\mathrm{K}^{+}$mesons have an important role to play.

\footnotetext{
1. Aubert. J.J. et al. Phys. Lett. B123. 275 (1983).
2. Close. F.E.. Roberts. R.G. \& Ross. G. G. Phys. Lett B129, 346 (1983)

. Siegel, P.B.. Kaufmann W.B. \& Gibbs. W.R. Phys. Rev. C31, 2184 (1985)

4. Murlow, D. et al. Phys, Rev. C25. 2619 (1982)

Brown, G.E., Dover, C.B., Siegel. P.B. \& Weise. W. Phys. Rev. Lett. 60, 2723 (1988)

6. Nambu, Y.\& Jona-Lasinio. G. Phys. Rev. 122, 345 (1961)

6. Nambu, Y. \& Jona-Lasinio. G. Phys. Re1. $122,345(1961)$ Phys. Lett. B200. 413 (1988)

8. Dunne. G.V.\& Thomas. A.W. Phys. Rev. D33, $2(61$ (1986)

9. Sick. I. Nucl. Phys. A434, 677 (1985)

10. Thomas, A.W. Prog. Part. nucl. Phys. 20, 21 (1988)

11. Guichon, P.A.M. Phys. Lett. B200. 235 (1988).
}

A.W. Thomas is in the Department of Physics and Mathematical Physics, The University of Adelaide, GPO Box 498, Adelaide, South Australia 5001 and J.E. Thomas is in the School of Physical Sciences, South Australian College of Advanced Education, Adelaide, South Australia 5000, Australia.
Daedalus

\section{Deep green power}

THE greenhouse effect is self-accelerating. The hotter the climate, the more power will be demanded for air conditioning, and the more carbon dioxide from fossil-fuel power stations will be poured into the atmosphere to heat up the greenhouse. Daedalus points out that carbon dioxide is readily soluble in water, especially under pressure. Water is also an ideal medium for dissipating the other waste product of power generation, low-grade heat. Because a great deal of fossil fuel, in the form of oil, natural gas and possibly beds of crystalline oceanic methane hydrate, is also located under water, Daedalus now puts forward the obvious answer. He is designing a submarine power station.

Many of the design problems have already been solved by the offshore oil rig and marine gas-turbine industries. But two fundamental problems need attention: how to get the air down to burn the deepsea fuel; and how to get the resulting electricity ashore.

A big gill to extract oxygen from the water itself is probably impractical. Air will have to be drawn from the surface down a bulky pipe, preferably fitted with a sequence of compressors to balance the pressures inside and outside the pipe as it descends into the high-pressure depths. It will then be mixed with fuel and burnt in the gas turbine; the exhaust gas will simply be released into the deep, where its carbon dioxide will dissolve.

To get the electricity ashore, Daedalus boldly proposes to use the conductivity of sea water itself. The output winding of his unit will simply be a plastic tube full of sea water, with one end opened to the ocean. The pipe would be laid all the way to shore, where it would form the primary winding of the distribution transformer. Its other end would return to the ocean, completing the watery circuit. Thus the a.c. from the alternator would be carried between ocean and shore transformers by a complete loop of sea water. No electrodes would be needed, and no power would be wasted in electrolytic effects. The diameter of the currentcarrying pipe could easily be made large enough to compensate for the relatively low conductivity of sea water.

Daedalus will give his power station neutral buoyancy, matching the neutral buoyancy of its plastic-pipe 'umbilical'. Thus it will be mobile. When one naturalgas borehole gives out, it will be able to move on to the next. It will also be able to 'browse' continuously over an ocean-floor field of methane hydrate, using its waste heat to disengage the weakly-bound methane. With no environmental impact at all, it will be the ultimately virtuous source of power.

David Jones 